\title{
EXPONENTIAL SUMS OVER PRIMES IN AN ARITHMETIC PROGRESSION
}

\author{
ANTAL BALOG AND ALBERTO PERELLI ${ }^{1}$
}

\begin{abstract}
In 1979 A. F. Lavrik obtained some estimates for exponential sums over primes in arithmetic progressions by an analytic method. In the present paper we give an estimate for the same sums, comparable with Lavrik's estimate, by means of elementary methods like Vaughan's identity.
\end{abstract}

1. In [2] A. F. Lavrik investigated the sum

$$
S(\alpha)=\sum_{\substack{n \leq N \\ n \equiv f(\bmod d)}} \Lambda(n) e(n \alpha) \quad\left(e(x)=e^{2 \pi i x}\right),
$$

where $N \geq 1,1 \leq f<d,(f, d)=1$. His main theorem was

ThEOREM A (A. F. LAVRIK, 1979). Let $S(\alpha)$ be defined by (1) with $\mid \alpha-$ $a / q \mid \leq 2 / N,(a, q)=1$ and $h=(q, d)$. Then

$$
S(\alpha) \ll\left(h N / d q^{1 / 2}+q^{1 / 2} N^{1 / 2}+(h / d)^{2 / 7} q^{3 / 14} N^{5 / 7}\right) \log ^{18} N .
$$

He also derived three corollaries from this theorem, concerning estimates for $S(\alpha)$ of the form (2) but with slightly different assumptions on $\alpha, q$ and $d$. His proof, as the title reveals, is based on analytic methods, mainly on density theorems for the zeros of Dirichlet's $L$-functions.

In the present note we show that a result of the type (2) may be obtained by using only simple elementary arguments like Vaughan's identity and the following well-known estimates.

LEMMA A.

$$
\sum_{\substack{x<m \leq x^{\prime} \\ m \equiv f(\bmod d)}} e(m \theta) \ll \min \left(\frac{x^{\prime}}{d}+1,\|\theta d\|^{-1}\right) .
$$

LEMMA B. If $X, Y, a, q \geq 1$ are integers and $(a, q)=1$, then

$$
\sum_{m \leq X} \min \left(Y,\left\|\frac{a m}{q}\right\|^{-1}\right) \ll \frac{X Y}{q}+(X+q) \log q
$$

(here $\|x\|=\min _{t \in \mathbf{Z}}|t-x|$ ).

Lemma A follows at once from the summation formula for the geometrical series. For the proof of Lemma B see, for example, [3].

Received by the editors November 29, 1983 and, in revised form, April 12, 1984.

1980 Mathematics Subject Classification. Primary 10G10.

Key words and phrases. Exponential sums over primes.

${ }^{1}$ The present paper was written when the first named author was a C.N.R. Visiting Professor at the University of Genova. 
We prove the following

ThEOREM. Let $S(\alpha)$ be defined by (1), with $|\alpha-a / q| \leq 2 / N,(a, q)=1$ and $h=(q, d)$. Then

$$
S(\alpha) \ll\left(\frac{h N}{d q^{1 / 2}}+\frac{q^{1 / 2} N^{1 / 2}}{h^{1 / 2}}+\frac{N^{4 / 5}}{d^{2 / 5}}\right) \log ^{3} N
$$

This result is comparable with (2) and, of course, the corresponding corollaries can be derived.

The proof of our theorem is essentially the adaptation of Vaughan's method [4], which was worked out for the complete sum $\sum_{n<N} \Lambda(n) e(n \alpha)$. However, we remark that when $\alpha=a / q$ and $d=1$ the inequality (5) is stronger than Vaughan's [4] by a factor of $\log ^{1 / 2} N$. This slight improvement comes from the slightly different identity (see (8)) we use.

2. First of all we note that it is enough to prove (5) for $\alpha=a / q$; the more general result follows by a standard partial summation.

We may assume that

$$
N \geq d^{3}, \quad N \geq q
$$

otherwise (5) is a consequence of the trivial bound

$$
S(\alpha) \leq N / d+1
$$

We now use Vaughan's identity in the form given by Balog $[\mathbf{1}]$. Let $U$ be a parameter to be chosen later, satisfying $1 \leq U \leq N^{1 / 2}$ and define

$$
F(s)=\sum_{n \leq U} \Lambda(n) n^{-s}
$$

and

$$
M(s)=\sum_{n \leq U} \mu(n) n^{-s}
$$

Then

$$
\begin{aligned}
-\frac{\varsigma^{\prime}}{\zeta}(s)= & F(s)-\varsigma^{\prime}(s) M(s)-\varsigma(s) F(s) M(s) \\
& +\left(\frac{1}{\zeta}(s)-M(s)\right)\left(-\varsigma^{\prime}(s)-F(s) \zeta(s)\right)
\end{aligned}
$$


By comparing the coefficient of $n^{-s}$ on both sides we obtain

$$
\begin{aligned}
& \sum_{\substack{n \leq N \\
n \equiv f(\bmod d)}} \Lambda(n) e\left(\frac{a n}{q}\right)=\sum_{\substack{n \leq U \\
n \equiv f(\bmod d)}} \Lambda(n) e\left(\frac{a n}{q}\right) \\
& +\sum_{\substack{m n \leq N \\
n \leq U}} \sum_{\substack{ \\
m}} \mu(n)(\log m) e\left(\frac{a m n}{q}\right) \\
& m n \equiv f(\bmod d) \\
& -\sum_{\substack{m n \leq N \\
m \leq U^{2} \\
m n \equiv f(\bmod d)}} a_{m} e\left(\frac{a m n}{q}\right) \\
& +\sum_{\substack{m n \leq N \\
m, n>U \\
m n \equiv f(\bmod d)}} \mu(m) b_{n} e\left(\frac{a m n}{q}\right),
\end{aligned}
$$

where

$$
a_{m}=\sum_{\substack{m=r t \\ r t \leq U}} \mu(r) \Lambda(t), \quad b_{n}=\sum_{\substack{n=r t \\ t>U}} \Lambda(t)
$$

The optimal choice of $U$ is

$$
U=N^{2 / 5} / d^{1 / 5}
$$

Now we show that if $\left|a_{m}\right| \leq 1$ are arbitrary complex numbers and $M d \leq N$, then

$$
\sum_{\substack{m n \leq N \\ M<m \leq 2 M \\ m n \equiv f(\bmod d)}} a_{m} e\left(\frac{a m n}{q}\right) \ll \frac{h N}{d q}+\left(M+\frac{q}{h}\right) \log \frac{q}{h} .
$$

Indeed, the left-hand side of (12) is

$$
\begin{aligned}
& \ll \sum_{\substack{M<m \leq 2 M \\
(m, d)=1}}\left|\sum_{\substack{n \leq N / m \\
n \equiv f \bar{m}(\bmod d)}} e\left(\frac{a m n}{q}\right)\right| \\
& \ll \sum_{M<m \leq 2 M} \min \left(\frac{N}{M d},\left\|\frac{a d m}{q}\right\|^{-1}\right)
\end{aligned}
$$

by Lemma $\mathrm{A}(\bar{m}$ is defined by $m \bar{m} \equiv 1(\bmod d))$. Using $(d, q)=h$ and Lemma B we get (12). 
Next we show that if $\left|a_{m}\right| \leq 1$ and $\left|b_{n}\right| \leq 1$ are arbitrary complex numbers and $d \leq M \leq N / d$, then

$$
\begin{aligned}
\sum_{\substack{m n \leq N \\
M<m \leq 2 M \\
m n \equiv f(\bmod d)}} a_{m} b_{n} e\left(\frac{a m n}{q}\right) & \\
& \ll \frac{M^{1 / 2} N^{1 / 2}}{d^{1 / 2}}+\frac{h N}{d q^{1 / 2}}+\left(\frac{N}{M^{1 / 2} d^{1 / 2}}+\frac{N^{1 / 2} q^{1 / 2}}{h^{1 / 2}}\right) \log ^{1 / 2} \frac{q}{h} .
\end{aligned}
$$

Indeed, denoting the left-hand side of (13) by $R$ it is clear that

$$
R=\sum_{\substack{f_{1} f_{2} \equiv f(\bmod d) \\\left(f_{1}, d\right)=\left(f_{2}, d\right)=1}} R_{f_{1}, f_{2}} \ll d \sum_{\substack{f_{1} f_{2} \equiv f(\bmod d) \\\left(f_{1}, d\right)=\left(f_{2}, d\right)=1}}\left|R_{f_{1}, f_{2}}\right|,
$$

where

$$
R_{f_{1}, f_{2}}=\sum_{\substack{m n \leq N \\ M<m \leq 2 M \\ m \equiv f_{1}(\bmod d) \\ n \equiv f_{2}(\bmod d)}} a_{m} b_{n} e\left(\frac{a m n}{q}\right) .
$$

Using the Cauchy-Schwarz inequality we get

$$
\begin{aligned}
& \left|R_{f_{1}, f_{2}}\right|^{2} \ll \frac{M}{d}\left(\sum_{\substack{M<m \leq 2 M \\
m \equiv f_{1}(\bmod d)}}\left|\sum_{\substack{n \leq N / m \\
n \equiv f_{2}(\bmod d)}} b_{n} e\left(\frac{a m n}{q}\right)\right|^{2}\right) \\
& \ll \frac{M}{d}\left(\sum_{\substack{n, n^{\prime} \leq N / M \\
n \equiv n^{\prime} \equiv f_{2}(\bmod d)}} e\left(\sum_{\substack{M<m \leq 2 M \\
m \leq \min \left(N / n, N / n^{\prime}\right) \\
m \equiv f_{1}(\bmod d)}} e\left(\frac{a m\left(n-n^{\prime}\right)}{q}\right)\right)\right) \\
& \ll \frac{M}{d}\left(\frac{N}{d^{2}}+\frac{N}{M d} \sum_{k \leq N /(M d)} \min \left(\frac{M}{d},\left\|\frac{a d^{2} k}{q}\right\|^{-1}\right)\right)
\end{aligned}
$$

by Lemma A, and using $h \leq\left(d^{2}, q\right) \leq h^{2}$, Lemma B gives (13).

From (9), (12) and (13) we have

$$
S\left(\frac{a}{q}\right) \ll\left(\frac{h N}{d q^{1 / 2}}+\frac{N^{1 / 2} q^{1 / 2}}{h^{1 / 2}}+\frac{q}{h}+U^{2}+\frac{N}{d^{1 / 2} U^{1 / 2}}\right) \log ^{3} N
$$

provided that $U^{2} \leq N / d, U \geq d$. It is easy to see that the optimal choice of $U$ is (11) and this choice satisfies the above conditions, provided (6) holds. Finally we note that the term $q / h$ in the right-hand side of $(16)$ is majorized by $(N q)^{1 / 2} / h^{1 / 2}$. This completes the proof.

We wish to express our thanks to the referee for his suggestions. 


\section{REFERENCES}

1. A. Balog, On sums over primes, Banach Center Publ., Vol. 17 (to appear).

2. A. F. Lavrik, An analytic method of estimating trigonometric sums over primes in an arithmetic progression, Dokl. Akad. Nauk SSSR 248 (1979), no. 5; English transl., Soviet Math. Dokl. 20 (1979), 1121-1124.

3. R. C. Vaughan, On the estimation of trigonometrical sums over primes, and related questions, MittagLeffler Institut Report, no. 9, 1977.

4. _ Sommes trigonométriques sur les nombres premiers, C. R. Acad. Sci. Paris Sér. A 285 (1977), 981-983.

Mathematical institute of the Hungarian ACAdemy of Sciences, ReáltaNODA U. 13-15, 1053 BUDAPEST, HUNGARY

Istituto di Matematica, Via L. B. Alberti 4, 16132 Genova, Italy 